\title{
ANTIPHOSPHOLIPID ANTIBODY (APLA) SYNDROME: ADRENAL INSUFFICIENCY
}

\author{
Dr Akshatha Taranath Kamath¹, Dr Sandeep², Dr Gayathri Gopalakrishnan², \\ Dr Sharath Damodar ${ }^{3}$ and Dr Sampath Satish Kumar ${ }^{1}$ \\ Department of Endocrinology ${ }^{1}$, Medical Gastroenterology ${ }^{2}$ and Hematology ${ }^{3}$ \\ Narayana Hrudayalaya Hospitals, NH Health City, Bommasandra Industrial \\ Area, Anekal Taluk, Bangalore- 560099, India
}

\section{INTRODUCTION}

Antiphospholipid antibody (APLA) syndrome is a rare autoimmune systemic disorder which can occur as a primary condition or secondary to connective tissue diseases, most frequently Systemic Lupus Erythematosis. We are presenting a rare case of a lady who developed adrenal insufficiency secondary to adrenal vein thrombosis, as a late sequel to APLA Syndrome.

\section{CASE REPORT}

A 50yr-old lady admitted to the medical ICU with one day's history of severe abdominal pain and vomiting. She had previously been daignosed with APLA syndrome. She gave history of recurrent intrauterine deaths (5) and 6 abortions in the past. She was not on any regular medications except OCP which was started a month prior to her hospital admission. Investigations revealed Serum Sodium 134 (135-150)mEq/L, Potassium 3.7(3.55.0)mEq/L, Cortisol 20.14(0.49-58.60)ug/dl, APTT 30.6(26.1-33.3)sec, PT 11.7(11.0-15.0)sec, D-Dimer $\quad 0.2(0.0-0.3) \mathrm{mg} / \mathrm{l}$. CT scan showed bilateral adrenal gland and retroperitoneal inflammation. In keeping with past history of APLA syndrome, bilateral adrenal vein thrombosis was considered and she was started on LMW Heparin.

Once stable, she was started on oral anticoagulant and discharged. Three weeks later, she visited the OPD with tiredness, dizziness and an episode of collapse. BP was $110 / 70$ and $120 / 80 \mathrm{mmHg}$ on sitting and standing respectively. Because of recent history of adrenal vein thrombosis, 8AM Serum Cortisol was done, which was found to be $5.38 \mathrm{ug} / \mathrm{dl}$. $250 \mathrm{mcg}$ ACTH stimulation test revealed baseline cortisol of $2.98 \mathrm{ug} / \mathrm{dl}$ and '30'minute cortisol post ACTH of $5.49 \mathrm{ug} / \mathrm{dl}$. She was diagnosed with adrenal insufficiency as a delayed complication of adrenal vein thrombosis. She was started on Tablet Hydrocortisone 10mg$5 \mathrm{mg}-5 \mathrm{mg}$. Recent ACTH Stimulation test did not show improvement in adrenal response.
She has been on maintenance dose of Hydrocortisone.

\section{DISCUSSION}

The characteristic feature of APLA Syndrome is recurrent arterial and venous thrombosis. However, adrenal vein thrombosis and adrenal insufficiency are relatively rare. OCP along with lack of anticoagulation therapy might have precipitated adrenal vein thrombosis. Even though patient did not have adrenal insufficiency acutely, she developed the condition as a delayed complication.

\section{CONCLUSION}

Adrenal vein thrombosis leads to increase in adrenal venous pressure leading to haemorrhagic necrosis of the gland. Stress, severe systemic illness, previous thromboembolic disease and post- surgery state are some of the risk factors for adrenal haemorrhage. Patients who are at risk of developing thromboembolic diseases and have not received anticoagulation therapy found to be at high risk of developing this complication as seen in our patient.

Treating physicians should have high index of suspicion regarding rare complications and monitor them regularly so that life threatening consequences can be avoided.

\section{REFERENCES}

1.Atanassova PA. Antiphospholipid syndrome and vascular ischemic (occlusive) diseases: an overview. Yonsei Med J 2007; 48: 901-26

2.Presotto F, Fornasini F, Betterle C, Federspil G, Rossato $M$. Acute adrenal failure as the heralding symptom of primary antiphospholipid syndrome: report of a case and review of the literature. Eur J Endocrinol. 2005;153(4):50714. doi: $10.1530 /$ eje.1.02002.

3. Fujishima N, Komatsuda A, Ohyagi H, Fujishima M, Tada $\mathrm{M}$, Ohtani $\mathrm{H}$, et al. Adrenal insufficiency complicated with antiphospholipid syndrome (APS) Intern Med. 2006;45(16):963-6.

4. Fischer, M. J., Rauch, J., and Levine, J. S. (2007). The antiphospholipid syndrome. Annals of the Rheumatic Diseases 10, 136-142 